\title{
Development of a luciferase-based reporter of transcriptional gene silencing that enables bidirectional mutant screening in Arabidopsis thaliana
}

So Youn Won ${ }^{1}$, Shengben Li ${ }^{1}$, Binglian Zheng ${ }^{1,7}$, Yuanyuan Zhao ${ }^{1}$, Dongming Li ${ }^{1,2}$, Xin Zhao ${ }^{1,3}$, Huilan Yi ${ }^{1,4}$, Lei Gao', Thanh Theresa Dinh ${ }^{1,5}$ and Xuemei Chen ${ }^{1,6^{*}}$

\begin{abstract}
Background: Cytosine methylation is an important chromatin modification that maintains genome integrity and regulates gene expression through transcriptional gene silencing. Major players in de novo methylation guided by siRNAs (known as RNA-directed DNA methylation, or RdDM), maintenance methylation, and active demethylation have been identified in Arabidopsis. However, active demethylation only occurs at a subset of RdDM loci, raising the question of how the homeostasis of DNA methylation is achieved at most RdDM loci. To identify factors that regulate the levels of cytosine methylation, we aimed to establish a transgenic reporter system that allows for forward genetic screens in Arabidopsis.
\end{abstract}

Results: We introduced a dual $35 \mathrm{~S}$ promoter (d35S) driven luciferase reporter, LUCH, into Arabidopsis and isolated a line with a moderate level of luciferase activity. LUCH produced transgene-specific 24 nucleotide siRNAs and its d35S contained methylated cytosine in CG, CHG and CHH contexts. Treatment of the transgenic line with an inhibitor of cytosine methylation de-repressed luciferase activity. Mutations in several components of the RdDM pathway but not the maintenance methylation genes resulted in reduced $d 355$ methylation, especially $\mathrm{CHH}$ methylation, and de-repression of luciferase activity. A mutation in MOM1, which is known to cooperate with RdDM to silence transposons, reduced d35S DNA methylation and de-repressed LUCH expression. A mutation in ROS1, a cytosine demethylation enzyme, increased d35S methylation and reduced LUCH expression.

Conclusion: We developed a luciferase-based reporter, LUCH, which reports both DNA methylation directed by small RNAs and active demethylation by ROS1 in Arabidopsis. The moderate basal level of LUCH expression allows for bi-directional genetic screens that dissect the mechanisms of DNA methylation as well as demethylation.

Keywords: Cytosine methylation, Demethylation, RdDM, MOM1, Transcriptional gene silencing, siRNA

\section{Background}

Cytosine methylation is a major epigenetic mechanism that establishes transcriptional gene silencing (TGS) to maintain genome integrity and regulate gene expression in plants and mammals (reviewed in [1]). Well-known biological phenomena involving DNA methylation as an

\footnotetext{
* Correspondence: xuemei.chen@ucr.edu

'Department of Botany and Plant Sciences, Institute of Integrative Genome

Biology, University of California, Riverside, CA 92521, USA

${ }^{6}$ Howard Hughes Medical Institute, University of California, Riverside, CA 92521, USA

Full list of author information is available at the end of the article
}

underlying mechanism include imprinting, paramutation and $\mathrm{X}$ chromosome inactivation. In plants, transposons and repetitive elements are methylated, thereby keeping transposons silenced and immobilized and consequently protecting the genome from damage by these mobile elements. Also, when transposons or repeats are located in the regulatory regions of genes, DNA methylation at the transposons or repeats may influence the transcription of the nearby genes through TGS.

The enzymes that initiate, maintain, and erase DNA methylation in Arabidopsis have been identified and

\section{() Biomed Central}


characterized (reviewed in [1]). De novo DNA methylation, also known as RNA-directed DNA methylation (RdDM), requires DOMAIN REARRANGED METHYLTRANSFERASE2 (DRM2), which is guided to specific genomic loci by 24 nucleotide small interfering RNAs (siRNAs). siRNAs are synthesized from repeats and transposons in an RNA polymerase IV (Pol IV)-, RNA DEPENDENT RNA POLYMERASE2 (RDR2)-, and DICERLIKE3 (DCL3)-dependent manner. Pol IV is thought to transcribe these loci into single-stranded RNAs, which are then rendered double-stranded by RDR2. DCL3 dices the double-stranded RNAs into 24 nucleotide siRNAs, which are loaded into the ARGONAUTE4 (AGO4)-clade of AGO proteins (reviewed in [2]). Base-pairing between the AGO4-loaded siRNAs and nascent transcripts produced by Pol $\mathrm{V}$ is thought to recruit AGO4/siRNAs and DRM2 to the RdDM targets, resulting in de novo methylation in a sequence-specific manner (reviewed in [2]). After the initial establishment of DNA methylation, hemimethylated cytosines in CG and CHG contexts resulting from DNA replication are fully methylated by METHYLTRANSFERASE1 (MET1) and CHROMOMETHYLTRANSFERASE3 (CMT3), respectively (reviewed in [1]). The positive feedback loop in which DNA methylation promotes siRNA biogenesis, which guides de novo DNA methylation, needs to be kept in check to prevent the expansion of heterochromatin and the sporadic silencing of genic regions. One such mechanism is DNA demethylation. Four DNA glycosylase/lyase enzymes remove methyl cytosine through a base excision repair mechanism (reviewed in [3]). DEMETER establishes imprinting during female gametogenesis and REPRESSOR OF SILENCING1 (ROS1), DEMETER-LIKE2 (DML2) and DML3 prevent hypermethylation in vegetative tissues.

Although the enzymes that deposit or erase DNA methylation are known, how these enzymes are regulated to achieve the proper homeostasis of DNA methylation is still nebulous. Although demethylation can keep DNA methylation in check, whole genome bisulfite sequencing in the ros $1 \mathrm{dml2} d m l 3$ triple mutant revealed that only a few hundred loci are hypermethylated [4] and are thus targets of demethylation. Since thousands of loci harbor DNA methylation, generate siRNAs and are targets of RdDM, it remains to be determined how most RdDM loci achieve homeostasis of DNA methylation. It is likely that other, as yet unknown, mechanisms prevent the hypermethylation of RdDM loci.

In addition to the RdDM pathway, MORPHEUS' MOLECULE1 (MOM1) impacts TGS in a complex manner usually without affecting the levels of cytosine methylation at target loci [5-7]. It encodes a protein with similarities to chromatin remodeling ATPases and silences endogenous loci and transgenes by an unknown mechanism [7].
MOM1 exhibits a complex relationship with RdDM depending on the target loci [5]. It functions either in the same pathway as RdDM or in a parallel pathway, or it could even antagonize the silencing by $\mathrm{RdDM}$. Some loci are transcriptionally suppressed by MOM1 independently of RdDM.

Forward genetic screens in Arabidopsis can help reveal mechanisms that regulate DNA methylation. In fact, most of the currently known genes involved in DNA methylation or demethylation were uncovered through genetic screens. However, most prior genetic screens were based on the isolation of mutations that release $\mathrm{RdDM}$ to result in de-repressed reporter gene expression, thus precluding the identification of negative regulators of DNA methylation. So far, the only known negative factors in DNA methylation, ROS1 and ROS3 (a protein required for ROS1-mediated demethylation), were isolated from genetic screens using the RD29A:: $L U C$ transgene system [8,9]. Therefore, RD29A::LUC happens to be a target of ROS1-mediated demethylation. As mentioned above, the relatively lower number of ROS1/ DML2/DML3 target loci in the genome as compared to the number of RdDM loci suggests the presence of unknown negative factors for methylation acting independently of, or in combination with, active demethylation by ROS1/DML2/DML3. Consequently, it is valuable to develop additional RdDM reporter transgenes inserted into different genomic locations to allow for the identification of these negative players.

Here, we report the establishment of a firefly $L U C I$ FERASE (LUC)-based reporter transgene driven by a dual $35 \mathrm{~S}$ promoter that harbors DNA methylation in CG, CHG, and CHH contexts in Arabidopsis. We show that $L U C$ expression is repressed mainly through $\mathrm{CHH}$ methylation in an RdDM-dependent manner. MOM1 also plays a role in DNA methylation and TGS of the reporter. More importantly, the moderate level of basal LUC expression in wild-type plants allows for genetic screens that aim at the isolation of mutants with not only defective but also enhanced DNA methylation. In fact, a ros 1 allele with reduced transgene expression was isolated using this system. The reporter line will prove to be an effective tool in dissecting the mechanisms that regulate DNA methylation.

\section{Results and discussion}

\section{Generation of the luciferase reporter line, $L U C H$}

Initially, we aimed to establish a $L U C$-based transgene that reported both TGS by RdDM and post-transcriptional gene silencing by miRNAs to allow for forward genetic screens. A transgene was constructed such that $L U C$ was C-terminally fused in frame to the partial AP2 fragment containing the miR172 binding site [10] and the transgene was driven by a dual $35 S$ promoter, which will be referred 
to as d35S, from Cauliflower Mosaic Virus (d35S::LUC$A P 2)$. In the same vector, d35S-driven NEOMYCIN PHOSPHOTRANSFERASE II (d35S::NPTII) served as a selectable marker for plant transformation (Figure 1). This construct was introduced into the rna-dependent rna polymerase6-11 (rdr6-11) [11-13] mutant background to prevent sense transgene post-transcriptional silencing (SPTGS; [11-13]) and one line with moderate levels of LUC signal was isolated to enable bidirectional genetic screens based on higher or lower LUC signals. The d35S::LUCAP2 transgene in this line was named LUCH (LUC repressed by $\mathrm{CHH}$ methylation), as we found later that it was repressed by $\mathrm{CHH}$ methylation in $d 35 S$. $L U C H$ was a one-copy insertion at a single genomic locus according to Southern blot analysis using the $L U C$ sequence as a probe (Additional file 1: Figure S1). Thermal asymmetric interlaced PCR (TAIL-PCR) followed by sequencing revealed that the transgene resided 20 nucleotides before the stop codon of At3g07350, a gene of unknown function. This insertion did not cause any obvious morphological phenotypes.

\section{LUCH does not report miRNA activity}

Since $L U C H$ contained a miR172 binding site, we first investigated whether it was able to report miRNA activity. If it were repressed by miR172, we would expect mutations in miRNA biosynthesis genes (reviewed in [14]), such as DICERLIKE1 (DCL1), HYPONASTIC LEAVES1 (HYL1), and SERRATE (SE) to de-repress $L U C H$ expression. In the F2 population of $L U C H$ crossed to $d c l 1-7$, LUC luminescence was moderately increased in 12 out of 216 segregating seedlings (Additional file 1: Figure S2A). Since $L U C H$ and DCL1 are not linked, the small proportion of seedlings with the moderately high LUC luminescence was not consistent with $d c l 1-7$ being able to de-repress $L U C H$ expression. Indeed, genotyping confirmed that only one of the 12 was homozygous for $d c l 1-7$, and three of the 12 were homozygous for the wild-type DCL1 allele. Therefore, the moderate increase was likely due to inherent variations in $L U C H$ expression or other background mutations. hyll and se-1 mutations also failed to increase LUC luminescence [see Additional file 1: Figure S2B and $\mathrm{S} 2 \mathrm{C}]$. These results demonstrate that $L U C H$ was unable to report miRNA activities even though the LUC transcript contains a miRNA-binding site in the 3' UTR.

\section{LUCH is regulated by RdDM-mediated TGS}

To evaluate whether $L U C H$ was repressed by RdDMmediated TGS, we first examined whether $L U C H$ had the molecular characteristics associated with RdDM. When compared with other reporter systems (NOSpro and $\alpha$ 'pro $[15,16]), d 35 S$ is more than twice as long as those promoters but has a similar percentage of GC content. $d 35 S$ has a relatively high non-CG composition (23 CG, 19 CHG and $138 / 128 \mathrm{CHH}$ in forward/reverse strands), which was also observed in the $\alpha$ 'pro system that was reported to be more sensitive to the regulation by RdDM than NOSpro $[15,16]$. McrBC-PCR was conducted using primers that specifically amplified the $d 35 S$ in $L U C H$ instead of that in $d 35 S:: N P T I I$ to evaluate the DNA methylation status of the $L U C H$ transgene. The results showed that $d 35 S$ was methylated whereas the LUC coding region was not (Figure 2A). Bisulfite sequencing revealed the presence of DNA methylation in $\mathrm{CG}, \mathrm{CHG}$, and $\mathrm{CHH}$ contexts (Figure 2B). The levels of $\mathrm{CHH}$ methylation were $22 \%$, which was particularly high compared to other previously established reporter lines of RdDM. For example, the $c l k-s k$ line had 15\% CHH methylation in the SUPERMAN 5' region [17]; the RD29A::LUC line had $1 \%$ and $6 \% \mathrm{CHH}$ methylation in the $R D 29 A$ promoter in wild type and ros1, respectively [8]. Treatment of LUCH seedlings with 5-aza-2'-deoxycytidine, an inhibitor of cytosine methylation increased LUC luminescence and LUC transcript levels, indicating that cytosine methylation transcriptionally silenced $L U C H$ expression [see Additional file 1: Figure S3].

Next, since RdDM target loci produce siRNAs, we determined the accumulation of siRNAs from the $L U C H$ and $235 S:: N P T I I$ transgenes. Even though we did not artificially introduce any hairpin source of $d 35 S$-specific siRNAs, siRNAs were detected in the $L U C H$ line by northern blotting using a $d 35 S$-specific probe (Figure 2C). High throughput sequencing was conducted to examine the small RNAs from the transgenes in more detail. siRNAs mapping to both DNA strands of the two transgenes were found; and 22 nucleotide siRNAs were the most abundant small RNA species [see Additional file 1: Figure S4A and $\mathrm{S} 4 \mathrm{~B}]$. Even though $\mathrm{LUCH}$ was introduced into $r d r 6-11$ to

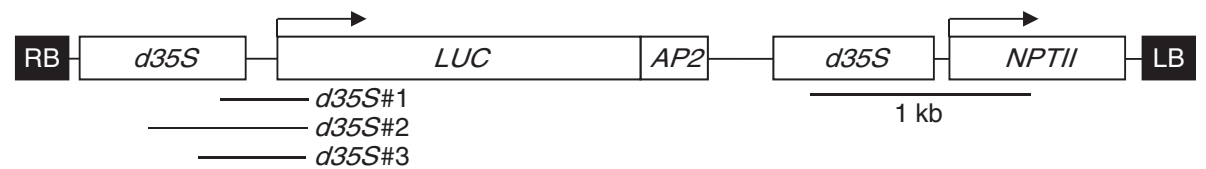

Figure 1 Structure of $\mathbf{L U C H}$ and its neighboring transgene. RB and LB, right border and left border of the T-DNA, respectively. The arrows

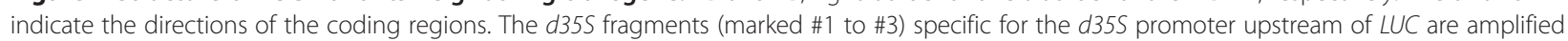
by PCR following digestion with the restriction enzyme McrBC as well as in bisulfite sequencing. 


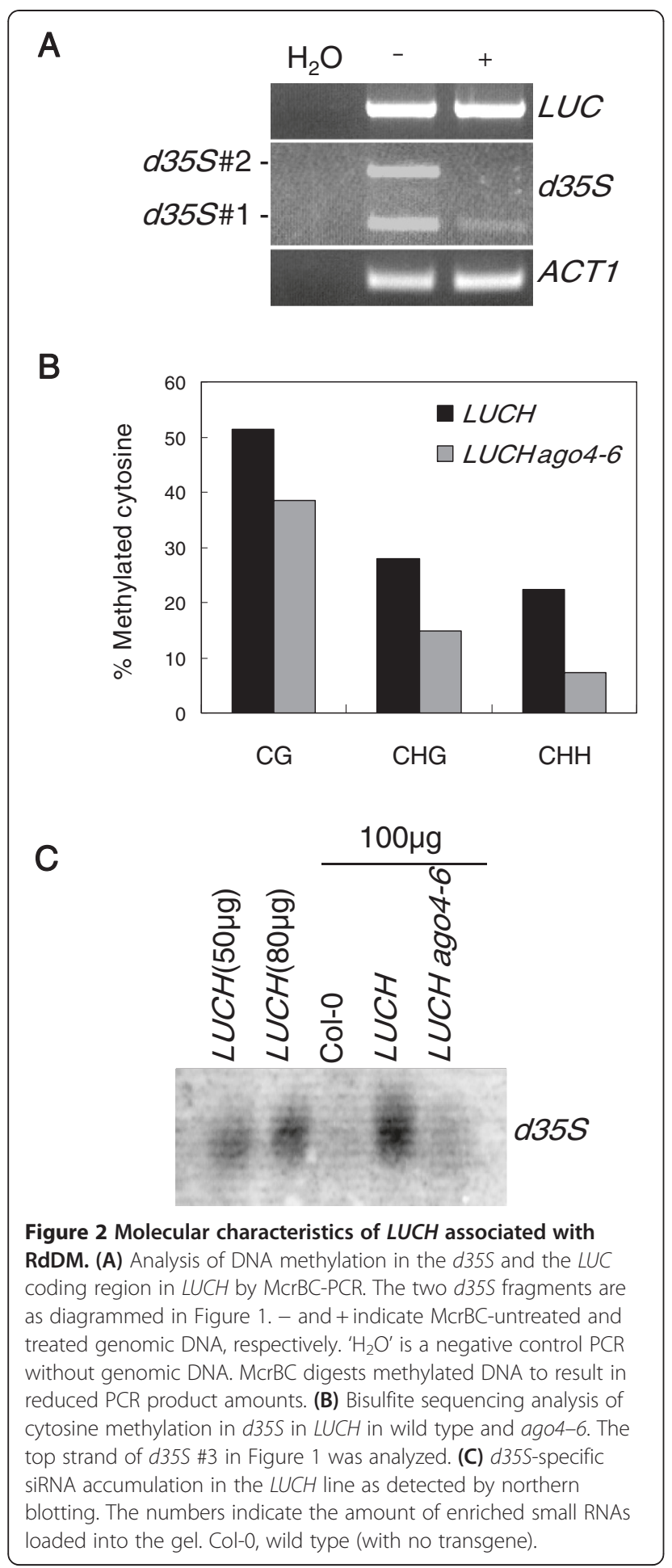

prevent S-PTGS by blocking the biogenesis of secondary siRNAs, 21 nucleotide and 22 nucleotide siRNAs mapping to the transgene were present, which suggests that PTGS was still occurring. Perhaps the siRNAs were primary siRNAs resulting from sense and antisense transcription from the locus or secondary siRNAs from the activities of
RDR2. Twenty four nucleotide siRNAs, which are associated with RdDM, were also present. Among 18 to $27 \mathrm{nu}-$ cleotide small RNAs that mapped to $d 35 S$ in $L U C H, 24$ nucleotide siRNAs accounted for approximately 19\% of the total [see Additional file 1: Figure S4A]. The $d 35 S$ promoters driving LUC and NPTII were 96\% identical in sequences. We took advantage of the sequence differences to determine whether both regions generated siRNAs. Indeed, siRNAs specific to each $d 35 S$ were found [see Additional file 1: Figure S4C; Additional file 2], indicating that each $d 35 S$ gave rise to siRNAs. The reverse strand $24 \mathrm{nu}-$ cleotide siRNAs were similar in quantity between the two transgenes (123 and 106 reads for $L U C H$ and $d 35 S:: N P T I I$, respectively). Interestingly, forward strand 24 nucleotide siRNAs were different in quantity between the two transgenes: 509 and 120 reads were from $d 35 S:: N P T I I$ and $L U C H$, respectively. The abundance of $d 35 S:: N P T I I-$ specific siRNAs was attributed to both higher diversity of siRNA species and higher levels of a subset of species [see Additional file 2]. The basis for the differential siRNA levels is unknown but may be due to differences in readthrough transcription at the two d35S. Taken together, LUCH exhibits the molecular characteristics associated with $\mathrm{RdDM}$, such as $\mathrm{CHH}$ methylation and 24 nucleotide siRNA production.

The regulation of $L U C H$ by $\mathrm{RdDM}$ was further supported by the fact that mutations in known RdDM pathway components de-repressed $L U C H$ expression. We mutagenized the $L U C H$ line with either ethyl methanesulfonate (EMS) or T-DNA and searched for mutants with higher LUC luminescence (Figure 3A). Genetic analyses demonstrated that each mutant with high LUC luminescence harbored a single, recessive mutation. Map-based cloning revealed that the mutations were in HUA ENHANCER1, AGO4, DRM2 and DEFECTIVE IN RNA-DIRECTED DNA METHYLATION1 (DRD1) [see Additional file 1: Figure S5], which are known genes in the RdDM pathway (reviewed in [1]). In addition, introducing nrpe1-1, a mutant of the largest subunit of Pol V (reviewed in [2]), into LUCH de-repressed LUC luminescence (Figure 3A). These mutants had higher levels of $L U C$ transcripts as revealed by RT-PCR (Figure 3B), indicating that the de-repression of $L U C H$ expression was at the transcriptional level. Since both LUC and NPTII are under the regulation of $d 35 S$, we analyzed the expression levels of NPTII by RT-PCR. The NPTII transcript levels were also increased in these RdDM mutants (Figure 3B). We next analyzed the DNA methylation status of $d 35 S$ in these mutants. Southern blot analysis with a $d 35 S$ specific probe showed that $d 35 S$-specific bands were downwardly shifted in ago4-6, drd1-12 and drm2-6 [see Additional file 1: Figure S6], indicating that DNA methylation at $d 35 S$ was reduced in ago4-6, drd1-12 and drm2-6. Bisulfite-sequencing with primers that allowed only amplification of the $d 35 S$ in $L U C H$ showed 


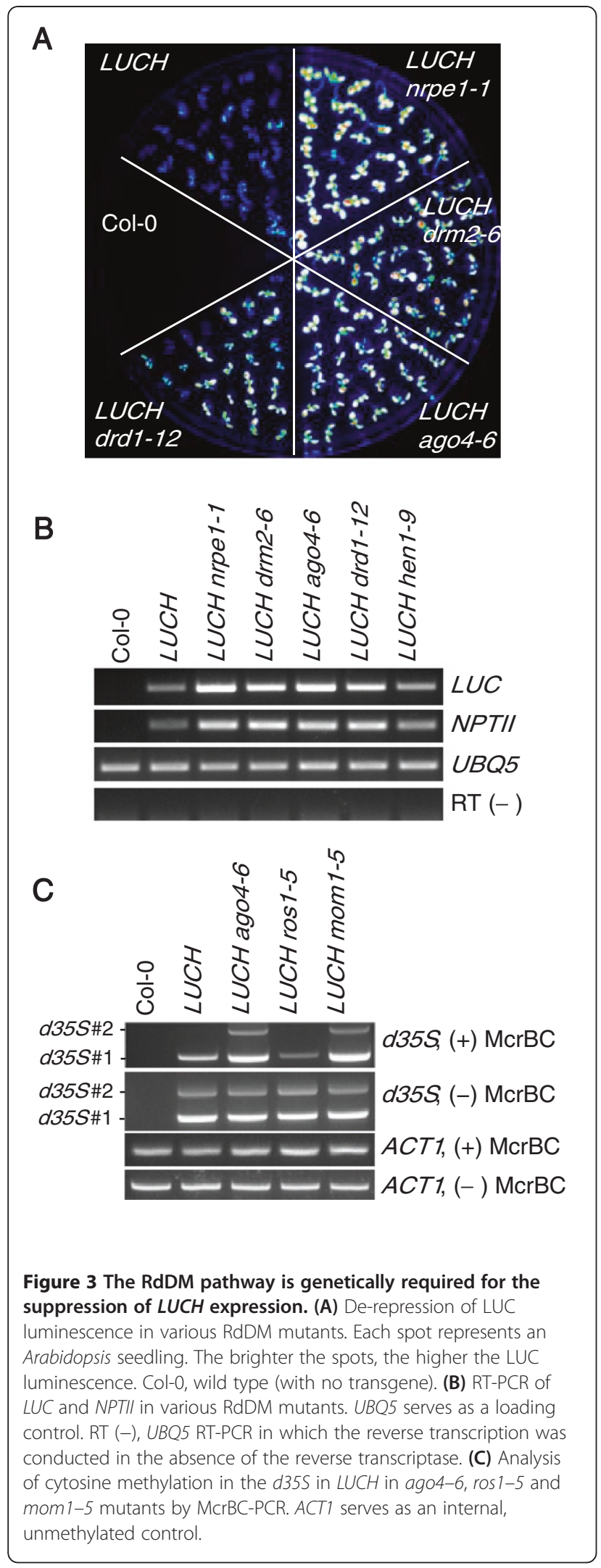

that the levels of DNA methylation were decreased in all sequence contexts in ago4-6, with $\mathrm{CHH}$ methylation being the most drastically decreased (Figure 2B). These results show that $\mathrm{LUCH}$ is repressed by de novo DNA methylation at $d 35 S$ and the repression requires $\mathrm{RdDM}$ components. To evaluate whether maintenance methylation at CG and CHG contexts by MET1 and CMT3, respectively, contributes to the repression of $L U C H$, we crossed met1-3 and cmt3-7 mutations into LUCH. met1-3 or $c m t 3-7$ did not affect $L U C H$ expression [see Additional file 1: Figure S7], indicating that this reporter line was mainly repressed by de novo methylation through DRM2. These molecular and genetic results demonstrate that $L U C H$ faithfully reports RdDMmediated TGS.

\section{LUCH is regulated by MOM1}

Our genetic screen also resulted in the isolation of a new mom1 allele (mom1-5) that displayed de-repressed LUC luminescence (Figure 4A; [see Additional file 1: Figure S5]). RT-PCR confirmed the increased levels of LUC and NPTII transcripts and the absence of MOM1 transcripts in the mutant (Figure 4B). DNA methylation at $235 S$ was moderately decreased in mom1-5, as revealed by McrBC-PCR and Southern blot analysis (Figure 3C; [see Additional file 1: Figure S6]). The reduction in DNA methylation in mom1-5 was less severe than in RdDM mutants [see Additional file 1: Figure S6]. Nonetheless, this shows that the DNA methylation and TGS of $L U C H$ require MOM1.

LUCH is regulated by ROS1-mediated DNA demethylation A major motivation to establish a $L U C$-based reporter was to enable the screening for mutants with enhanced silencing. The $\mathrm{LUCH}$ line, which exhibited a moderate basal level of LUC luminescence, was suitable for such a purpose. We performed T-DNA insertional mutagenesis
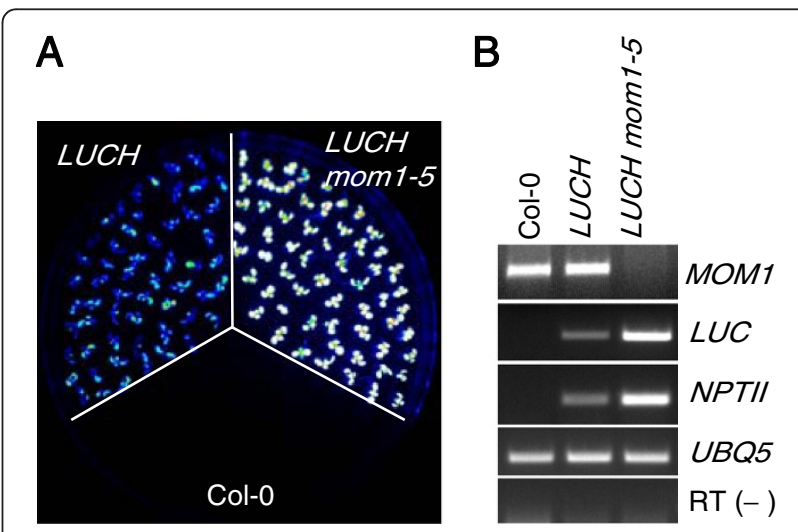

Figure $4 \mathrm{LUCH}$ is regulated by MOM1. (A) De-repression of LUC luminescence in LUCH mom 1-5. (B) RT-PCR of LUC, NPTII and MOM1 in wild type (Col-0), LUCH and LUCH mom 1-5. 


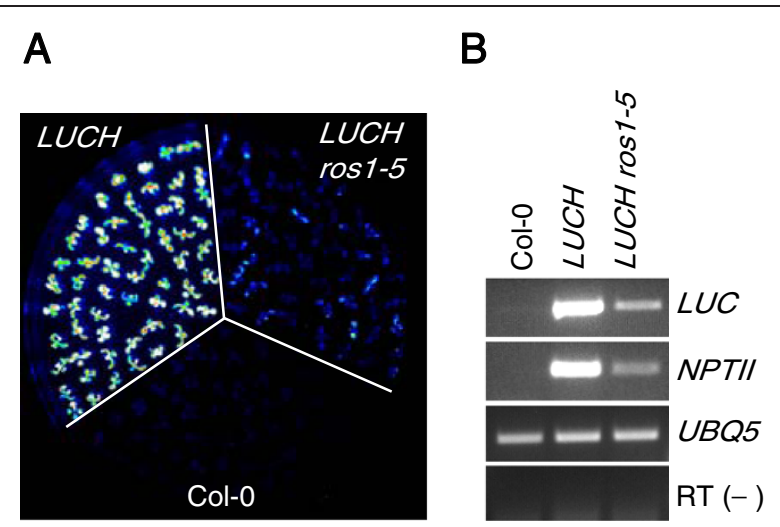

Figure 5 LUCH is targeted by ROS1-mediated DNA demethylation. (A) Reduction of LUC luminescence in LUCH ros 1-5. Note that the images were taken with the same exposure conditions as in Figures $3 \mathrm{~A}$ and $4 \mathrm{~A}$, but $L U C H$ appeared much brighter here because the contrast was adjusted to better reflect the differences between $\angle U C H$ and $L U C H$ ros 1-5. (B) RT-PCR of $L U C$ and NPTIl in wild type (Col-0), LUCH and LUCH ros $1-5$.

of the $L U C H$ line and isolated a recessive mutant allele with lower levels of LUC luminescence (Figure 5A). Map-based cloning identified this mutant as a new allele of ROS1 [see Additional file 1: Figure S5], a gene required for DNA demethylation. This suggested that loss of demethylation resulted in the accumulation of cytosine methylation in $d 35 S$ and reinforcement of TGS of $L U C H$. Indeed, there was an increase in DNA methylation of $d 35 S$ in $L U C H$ in ros1-5 according to McrBCPCR (Figure $3 C$ ). Levels of LUC and NPTII transcripts were decreased as determined by RT-PCR (Figure 5B). In addition, treatment of $L U C H$ ros $1-5$ seedlings with 5-aza-2'-deoxycytidine increased the expression of $\mathrm{LUCH}$ to wild-type levels [see Additional file 1: Figure S3], which further supported the notion that increased DNA methylation in ros1-5 led to enhanced TGS of $L U C H$. Therefore, even though $L U C H$ is transcriptionally repressed by $\mathrm{RdDM}$, the basal expression of $L U C H$ is relatively high such that the transgene can be used to screen for mutants with enhanced silencing.

\section{Conclusions}

We developed a transgenic LUC reporter system that reports both TGS by RdDM and MOM1, and ROS1mediated demethylation. Moderate expression of the reporter enables genetic screens in two directions to isolate mutants with decreased as well as increased DNA methylation. Considering that existing TGS reporter systems, such as the NOSpro, a'pro, and clk-sk lines, are mainly suitable for the isolation of positive players in RdDM, $L U C H$ is a useful genetic resource for the identification of negative players in $\mathrm{RdDM}$, for which nothing is known. Moreover, $\mathrm{LUCH}$ will potentially contribute to the better understanding of MOM1-mediated TGS or the mechanisms of active demethylation. For the latter, although RD29::LUC reports ROS1-mediated DNA demethylation, as a second reporter of ROS1-mediated demethylation residing at a different genomic location, $L U C H$ will enrich our resources to tackle the mechanisms of demethylation.

\section{Methods}

\section{Plant material}

Arabidopsis mutants used in this study were rdr6-11 [11], dcl1-7 [18], se-1 [19], hyl1 [20], met1-3 [21], cmt3-7 [17] and $d r d 3-1$ [22] and newly isolated drm2-6, ago4-6, drd1-12, hen1-9, ros1-5 and mom1-5. For map-based cloning of newly isolated mutants, LUCH $r d r 6-11$ in the Columbia-0 (Col-0) accession was introgressed into Landsberg erecta (Ler) by backcrossing to Ler five times and one line with a similar level of LUC activity as $\mathrm{LUCH}$ in Col-0 was isolated. The isolated mutants from $\mathrm{LUCH}$ rdr6-11 in Col were each crossed to LUCH rdr6-11 in Ler, and in the F2 population, seedlings with high (for drm2-6, ago4-6, drd1-12, hen1-9, and mom1-5) or low (ros1-5) luciferase activities were identified and served as the mapping population. Polymorphisms between Col-0 and Ler were utilized to map and clone the genes.

\section{Growth conditions and luciferase live imaging}

Arabidopsis thaliana seeds were surface-sterilized, planted on MS-agar plates containing $1 \%$ sucrose, and stratified at $4{ }^{\circ} \mathrm{C}$ for three days. Seedlings were grown at $23{ }^{\circ} \mathrm{C}$ under continuous lights for ten days. All experiments were performed with ten-day old seedlings unless otherwise specified. For luciferase live imaging, $1 \mathrm{mM}$ luciferin (a substrate of luciferase; Promega, Madison, Wisconsin, USA) in $0.01 \%$ Triton X-100 was sprayed onto the seedlings, which were then transferred to a Stanford Photonics Onyx Luminescence Dark Box. Luciferase images were taken with a Roper Pixis 1024B camera controlled by the WinView32 software at a two minute exposure time. Identical exposure conditions were used to capture all images in this study. The images were displayed and analyzed with WinView32 such that image contrast was adjusted to effectively distinguish the difference in intensities between different lines within a plate as previously described [23].

\section{Construction of transgene, southern blot analysis and TAIL-PCR}

The LUC coding region was amplified using the Rlucp1 and Rlucp2 primers and pRL-SV40 (Promega) as the template. $d 35 S:: L U C$ was constructed by replacing GFP in pAVA321 [24] with the LUC coding region using NcoI and $B a m H I$ restriction sites. The $d 35 S:: L U C$ cassette was cloned into the pPZP211 [25] at the SalI and BamHI restriction sites. An AP2 fragment including the miR172 
binding site was amplified from Col-0 genomic DNA with the primers AP2p26 and AP2p28 and inserted downstream of $d 35 S:: L U C$ in pPZP211 using BamHI and $E c o R$ I to generate $d 35 S:: L U C-A P 2$, which will be referred to as $L U C H$. The construct was introduced into rdr6-11 plants by Agrobacterium tumefaciens-mediated transformation. Southern blot analysis was performed according to the standard protocol [26] to evaluate the copy number of $L U C H$ using the full-length $L U C$ coding region as the probe. The probe was amplified with the primers lucp6 and lucp7, and radiolabeled with the RPN1633 Rediprime II Random Prime Labeling System (GE Healthcare Biosciences, Pittsburgh, Pennsylvania, USA). TAIL-PCR was performed as described [27]. Primers used are listed in Additional file 3: Table S1.

\section{Analysis of DNA cytosine methylation}

For the McrBC-PCR assay, two reactions were set up for each genomic DNA sample: McrBC-treated and untreated reactions. A total of $300 \mathrm{ng}$ genomic DNA was digested with 3 units of McrBC (New England Biolabs, Ipswich, Massachusetts, USA) for 25 minutes at $37^{\circ} \mathrm{C}$ in a $20 \mu \mathrm{l}$ reaction. Using $1 \mu \mathrm{l}(15 \mathrm{ng})$ of restricted genomic DNA as the template, genomic regions corresponding to $d 35 S$ or full length $L U C$ in the $L U C H$ transgene were amplified using the $35 \mathrm{Sf}$ and LUC $0.13 \mathrm{k} \mathrm{R}$ primers or the lucp6 and lucp7 primers, respectively. ACT1 was amplified with the Actin1-F and Actin1-R primers and used as a loading control. PCR products were analyzed on a $2 \%$ agarose gel stained with ethidium bromide. For Southern blot analysis, $15 \mu \mathrm{g}$ of genomic DNA was digested with AluI (NEB) and hybridization was performed following standard methods [28]. The $d 35 S$ promoter was PCR-amplified with the $35 \mathrm{Sf}$ and $35 \mathrm{Sr}$ primers and radiolabeled using the RPN1633 Rediprime II random prime labeling system (GE Healthcare). For bisulfite sequencing, $1 \mu \mathrm{g}$ of genomic DNA was subjected to bisulfite conversion using the EpiTect Bisulfite Kit according to the manufacturer's instructions (Qiagen, Hilden, Germany). Converted DNA was subjected to PCR reactions with primers YZ $35 \mathrm{~S}$ Bis F and YZ LUC Bis R and the PCR products were cloned into the pGEM-T Easy vector (Promega). At least 26 colonies were sequenced for each sample. Unique clones were obtained and analyzed for DNA methylation with Kithmeth (http://katahdin.mssm. edu/kismeth/revpage.pl) [28]. For 5-aza-2'-deoxycytidine (Sigma, St. Louis, Missouri, USA) treatment, seeds were germinated and grown on MS-agar medium containing $7 \mu \mathrm{g} / \mathrm{ml}$ of the chemical for two weeks and luciferase images were taken. Primers used are listed in Additional file 3: Table S1.

\section{Analysis of small RNA accumulation}

RNA isolation and hybridization to detect small RNAs were performed as described previously [29]. To detect
siRNAs from the $d 35 S$ promoter, a DNA fragment was amplified from the $d 35 S$ promoter using the $35 \mathrm{Sf}$ and $35 \mathrm{Sr}$ primers and cloned into the pGEM-T Easy vector (Promega). The plasmid was linearized by SpeI (NEB) and used as a template for in vitro transcription with T7 RNA polymerase (Promega) in the presence of $\left[\alpha-{ }^{32} \mathrm{P}\right]$ UTP. The labeled in vitro transcripts were used as the probe in northern blotting. Radioactive signals were detected with a Phosphorimager. For small RNA deep sequencing, a small RNA library was constructed using the TruSeq Small RNA Sample Prep Kit (Illumina, San Diego, California, USA) according to the manufacturer's instructions with some modifications. Instead of total RNA, 15 to 40 nucleotide long RNAs were used as the starting material. The small RNA library was sequenced by Illumina Hiseq2000 at the genomics core facility at the University of California Riverside. After the raw reads were filtered by the Illumina quality control pipeline and the adaptor sequences were trimmed, 14,363,865 reads between 18 nucleotides and 28 nucleotides were matched to the Arabidopsis genome (TAIRv10) as well as the transgenes with SOAP2 [30]. A total of 8,710,699 and 22,245 reads were mapped to the Arabidopsis genome and the transgenes, respectively, with no mismatches.

\section{RT-PCR}

cDNA was synthesized from $5 \mu \mathrm{g}$ of DNasel (Roche, Basel, Switzerland)-treated total RNA using Reverse Transcriptase (Fermentas, Burlington, Ontario, Canada) and oligo-dT (Fermentas) as the primer. Using cDNA and gene-specific primers, PCR was performed and RTPCR products were analyzed on a $2 \%$ agarose gel stained with ethidium bromide. The sequences of primers are listed in Additional file 3: Table S1.

\section{Additional files}

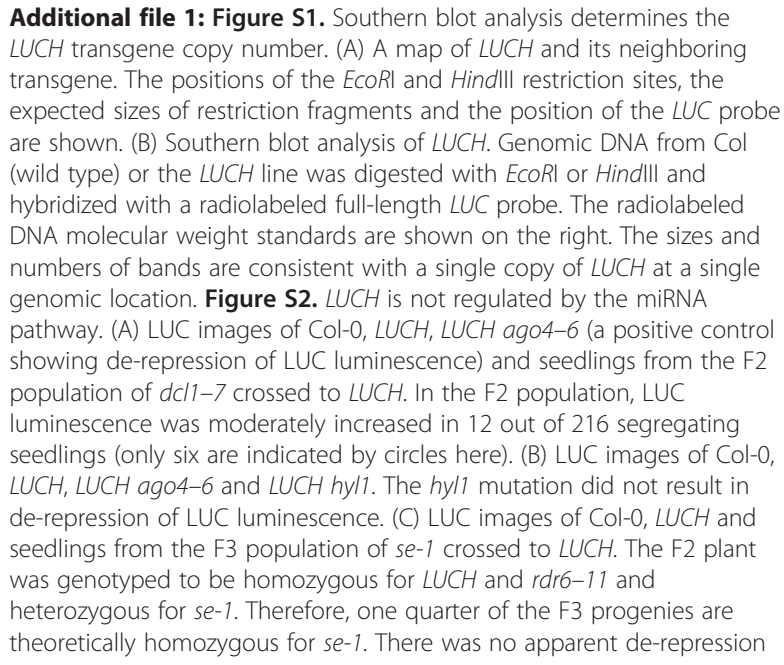


of $L U C H$ by se-1. Figure S3. De-repression of $L U C H$ and $L U C H$ ros $1-5$ by the methylation inhibitor 5-aza-2'-deoxycytidine (5Aza-dC). (A) Seedlings were grown on MS media for ten days (mock) or on $7 \mu \mathrm{g} / \mathrm{ml}$ 5Aza-dC-supplemented MS media for two weeks (5Aza-dC) followed by LUC luminescence imaging. (B) RT-PCR analysis of LUC and NPTII expression in mock- or 5Aza-dC-treated $L U C H$ and $L U C H$ ros $1-5$ seedlings. UBIQUITIN5 (UBQ5) was used as a loading control. The RT (-) reactions were performed with UBQ5 primers. Figure S4. Transgene-specific small RNAs in the LUCH line as determined by deep sequencing. (A) Size distribution of small RNAs mapping to the entire T-DNA containing LUCH and d35S::NPTII (total), the d35S promoter in LUCH (d35S) or the LUC coding sequence (LUC). (B) Distribution and abundance of 24 nucleotide small RNAs mapping to the LUCH and d35S::NPTII transgenes. Top and bottom figures indicate the distribution of 24 nucleotide siRNAs from forward and reverse strands, respectively. (C) Distribution and abundance of 24 nucleotide small RNAs that are specific to each $d 355$ promoter in the two transgenes. The $4 \%$ sequence variations between the $d 35 S$ in the two transgenes allowed the identification of these transgene-specific d35S siRNAs. Small RNAs mapping to both strands were detected. Figure S5. Schematic diagrams of the gene structures and the mutations in the newly isolated mutant alleles in this study. White and black rectangles indicate untranslated regions and coding exons, respectively. Lines represent introns. Arrows in mom 1-5 indicate the primers used for RT-PCR. The new hen1 allele is not diagrammed because the exact nature of the mutation is not known. The allele was shown by a genetic complementation test with known hen 1 mutants to be a new hen 1 allele. Figure S6. Southern blot analysis of cytosine methylation in $d 355$. (A) Map of the transgenes. Bars represent the probe, which should hybridize to both transgene promoters. (B) Genomic DNA was isolated from Col-0, LUCH, LUCH ago4-6 and LUCH drd1-12, digested with cytosine methylation-sensitive Alul and hybridized with the radiolabeled $d 355$ probe. DNA bands are shifted downward in ago4-6 and $\operatorname{drd1} 1-12$, indicating that DNA methylation in 1355 is decreased in ago4-6 and drd1-12. Though the juxtaposed lanes are discontinuous, they are from a single gel. The phosphor-image was taken from a single membrane. (C) Southern blot analysis of Col-0, LUCH, LUCH ago4-6, LUCH mom 1-5 and LUCH drm2-6. DNA bands are shifted downward to a lesser extent in mom1-5 than in ago4-6 or drm2-6. Figure S7. LUCH is not repressed by MET1 or CMT3. (A) LUC imaging of seedlings from an F3 population of cmt3-7 crossed to $L U C H$. The F2 plant was genotyped to be homozygous for $\mathrm{LUCH}$ and $r d r 6-11$ and heterozygous for $\mathrm{Cm} 33-7$. Therefore, one quarter of the $\mathrm{F} 3$ progenies are theoretically homozygous for $\mathrm{Cmt3-7}$. If CMT3 represses $L U C H$, de-repression of $L U C H$ is expected in one quarter of the seedlings. No such de-repression was observed. (B) LUC imaging of an F2 population of met $1-3$ crossed to $L U C H$. Seedlings with weakly de-repressed LUC signal were identified (circled), genotyped, and found not to be homozygous for met1-3. Note that MET1 and LUCH are not linked, such that $3 / 16$ of the seedlings are expected to be LUCH (or $\mathrm{LUCH} /+$ ) met1-3.

Additional file 2: Sequences of 24 nucleotide small RNAs mapping specifically to each $d 35 S$ in the two transgenes. Small RNAs were classified based on their transgene origin and strandedness. The sequences of the small RNAs, the number of reads and the positions of their $5^{\prime}$ nucleotides along the construct as shown in Additional file 1: Figure $\mathrm{S} 4$ is shown.

Additional file 3: Table S1 DNA oligonucleotides used in this study.

\section{Abbreviations}

d35S: dual $35 \mathrm{~S}$ promoter from Cauliflower mosaic virus; LUC: Luciferase; LUCH: LUC repressed by CHH methylation; RdDM: RNA-directed DNA methylation; RT-PCR: reverse transcriptase-polymerase chain reaction; siRNA: small interfering RNA; TAIL-PCR: thermal asymmetric interlaced PCR; TGS: transcriptional gene silencing; UTR: untranslated region.

\section{Competing interests}

The authors declare that they have no competing interests.

\section{Acknowledgments}

The work was supported by grants from the National Science Foundation (MCB-1021465) and the National Institutes of Health (GM061146) to X.C. The work was also supported by the Howard Hughes Medical Institute and the Gordon and Betty Moore Foundation. TD was supported by an NSF ChemGen IGERT program (DGE0504249). XZ was the recipient of a Chinese Academy of Sciences visiting scholar fund. HY was supported by funds from the China Scholarship Council.

\section{Author details}

${ }^{1}$ Department of Botany and Plant Sciences, Institute of Integrative Genome Biology, University of California, Riverside, CA 92521, USA. ${ }^{2}$ School of Life Sciences, Lanzhou University, Lanzhou 730000, Gansu, China. ${ }^{3}$ Laboratory of Plant Stress Ecophysiology and Biotechnology, Cold and Arid Regions Environmental and Engineering Research Institute, Chinese Academy of Sciences, 320 Donggang West Road, Lanzhou 730000, Gansu, China. ${ }^{4}$ School of Life Science, Shanxi University, 92 Wucheng Road, Taiyuan 030006, China. ${ }^{5}$ NSF ChemGen IGERT program, University of California, Riverside, CA 92521 USA. ${ }^{6}$ Howard Hughes Medical Institute, University of California, Riverside, CA 92521, USA. ${ }^{7}$ Current address: State Key Laboratory of Genetic Engineering and Institute of Plant Biology, School of Life Sciences, Fudan University, Shanghai 200433, China.

\section{Authors' contributions}

SYW and XC wrote the manuscript. SYW did the RT-PCR, McrBC-PCR, 5aza-dC treatments, the genetic analysis of $L U C H$, and identified two mutants to be drm2-6 and ros $1-5$ through map-based cloning. SL performed the siRNA

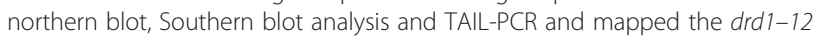
mutant. BZ transformed the reporter construct and selected the LUCH line. YZ mapped ago4-6. XZ mapped mom1-5 and performed Southern blot analysis. DL mapped hen 1-9 and constructed the small RNA library. HY did McrBC-PCR. LG analyzed the small RNA library data. TTD did bisulfite sequencing. XC constructed the reporter plasmid, conceived and guided the project. All authors read and approved the final manuscript.

Received: 1 March 2012 Accepted: 18 April 2012

Published: 7 June 2012

\section{References}

1. Law JA, Jacobsen SE: Establishing, maintaining and modifying DNA methylation patterns in plants and animals. Nat Rev Genet 2010, 11:204-220.

2. Haag JR, Pikaard CS: Multisubunit RNA polymerases IV and V: purveyors of non-coding RNA for plant gene silencing. Nat Rev Mol Cell Biol 2011, 12:483-492.

3. Furner IJ, Matzke M: Methylation and demethylation of the Arabidopsis genome. Curr Opin Plant Biol 2011, 14:137-141.

4. Lister R, O'Malley RC, Tonti-Filippini J, Gregory BD, Berry CC, Millar AH, Ecker $J R$ : Highly integrated single-base resolution maps of the epigenome in Arabidopsis. Cell 2008, 133:523-536.

5. Yokthongwattana C, Bucher E, Caikovski M, Vaillant I, Nicolet J, Mittelsten Scheid O, Paszkowski J: MOM1 and Pol-IV/V interactions regulate the intensity and specificity of transcriptional gene silencing. EMBO J 2010, 29:340-351.

6. Vaillant I, Schubert I, Tourmente S, Mathieu O: MOM1 mediates DNA-methylation-independent silencing of repetitive sequences in Arabidopsis. EMBO Rep 2006, 7:1273-1278.

7. Amedeo P, Habu Y, Afsar K, Mittelsten Scheid O, Paszkowski J: Disruption of the plant gene MOM releases transcriptional silencing of methylated genes. Nature 2000, 405:203-206.

8. Zheng X, Pontes O, Zhu J, Miki D, Zhang F, Li WX, lida K, Kapoor A, Pikaard CS, Zhu JK: ROS3 is an RNA-binding protein required for DNA demethylation in Arabidopsis. Nature 2008, 455:1259-1262.

9. Gong Z, Morales-Ruiz T, Ariza RR, Roldan-Arjona T, David L, Zhu JK: ROS1, a repressor of transcriptional gene silencing in Arabidopsis, encodes a DNA glycosylase/lyase. Cell 2002, 111:803-814.

10. Chen X: A microRNA as a translational repressor of APETALA2 in Arabidopsis flower development. Science 2004, 303:2022-2025.

11. Peragine A, Yoshikawa M, WU G, Albrecht HL, Poethig RS: SGS3 and SGS2/SDE1/ RDR6 are required for juvenile development and the production of transacting siRNAs in Arabidopsis. Genes Dev 2004, 18:2368-2379.

12. Mourrain $P$, Beclin C, Elmayan T, Feuerbach F, Godon C, Morel JB, Jouette D, Lacombe AM, Nikic S, Picault N, Rémoué K, Sanial M, Vo TA, Vaucheret H: 
Arabidopsis SGS2 and SGS3 genes are required for posttranscriptional gene silencing and natural virus resistance. Cell 2000, 101:533-542.

13. Dalmay T, Hamilton A, Rudd S, Angell S, Baulcombe DC: An RNA-dependent RNA polymerase gene in Arabidopsis is required for posttranscriptional gene silencing mediated by a transgene but not by a virus. Cell 2000, 101:543-553.

14. Chen X: Small RNAs and their roles in plant development. Annu Rev Cell Dev Biol 2009, 25:21-44.

15. Matzke M, Aufsatz W, Kanno T, Daxinger L, Papp I, Mette MF, Matzke AJ: Genetic analysis of RNA-mediated transcriptional gene silencing. Biochim Biophys Acta 2004, 1677:129-141.

16. Aufsatz W, Mette MF, Matzke AJ, Matzke M: The role of MET1 in RNA-directed de novo and maintenance methylation of $\mathrm{CG}$ dinucleotides. Plant Mol Biol 2004, 54:793-804.

17. Lindroth AM, Cao X, Jackson JP, Zilberman D, McCallum CM, Henikoff S, Jacobsen SE: Requirement of CHROMOMETHYLASE3 for maintenance of CpXpG methylation. Science 2001, 292:2077-2080.

18. Robinson-Beers K, Pruitt RE, Gasser CS: Ovule development in wild-type Arabidopsis and two female-sterile mutants. Plant Cell 1992, 4:1237-1249.

19. Prigge MJ, Wagner DR: The Arabidopsis SERRATE gene encodes a zinc-finger protein required for normal shoot development. Plant Cell 2001, 13:1263-1280.

20. Lu C, Fedoroff N: A mutation in the Arabidopsis HYL1 gene encoding a dsRNA binding protein affects responses to abscisic acid, auxin, and cytokinin. Plant Cell 2000, 12:2351-2366.

21. Saze H, Scheid OM, Paszkowski J: Maintenance of CpG methylation is essential for epigenetic inheritance during plant gametogenesis. Nat Genet 2003, 34:65-69.

22. Kanno T, Huettel B, Mette MF, Aufsatz W, Jaligot E, Daxinger L, Kreil DP, Matzke M, Matzke AJM: Atypical RNA polymerase subunits required for RNA-directed DNA methylation. Nat Genet 2005, 37:761-765.

23. Chinnusamy V, Stevenson B, Lee BH, Zhu JK: Screening for gene regulation mutants by bioluminescence imaging. Sci STKE 2002, 2002:pl10.

24. von Arnim AG, Deng XW, Stacey MG: Cloning vectors for the expression of green fluorescent protein fusion proteins in transgenic plants. Gene 1998, 221:35-43.

25. Hajdukiewicz P, Svab Z, Maliga P: The small, versatile pPZP family of Agrobacterium binary vectors for plant transformation. Plant Mol Biol 1994, 25:989-994.

26. Sambrook J, Fritsch EF, Maniatis T: Molecular Cloning: A Laboratory Manual. 2nd edition. Cold Spring Harbor, NY: Cold Spring Harbor Laboratory Press; 1989.

27. Liu Y-G, Chen Y: High-efficiency thermal asymmetric interlaced PCR for amplification of unknown flanking sequences. Biotechniques 2007, 43:649-656.

28. Gruntman E, Qi Y, Slotkin RK, Roeder T, Martienssen R, Sachidanandam R: Kismeth: analyzer of plant methylation states through bisulfite sequencing. BMC Bioinforma 2008, 9:371.

29. Park W, Li J, Song R, Messing J, Chen X: CARPEL FACTORY, a dicer homolog, and HEN1, a novel protein, act in microRNA metabolism in Arabidopsis thaliana. Curr Biol 2002, 12:1484-1495.

30. Li R, Yu C, Li Y, Lam T-W, Yiu S-M, Kristiansen K, Wang J: SOAP2: an improved ultrafast tool for short read alignment. Bioinformatics 2009, 25:1966-1967.

doi:10.1186/1758-907X-3-6

Cite this article as: Won et al: Development of a luciferase-based reporter of transcriptional gene silencing that enables bidirectional mutant screening in Arabidopsis thaliana. Silence 2012 3:6.

\section{Submit your next manuscript to BioMed Central and take full advantage of:}

- Convenient online submission

- Thorough peer review

- No space constraints or color figure charges

- Immediate publication on acceptance

- Inclusion in PubMed, CAS, Scopus and Google Scholar

- Research which is freely available for redistribution 